\title{
Division Algebras, Extended Supersymmetries and Applications
}

\author{
F. Toppan ${ }^{\mathrm{a}}$ \\ ${ }^{a}$ CCP - CBPF, Rua Dr. Xavier Sigaud 150, \\ cep 22290-180, Urca, Rio de Janeiro (RJ), Brazil
}

I present here some new results which make explicit the role of the division algebras $\mathbf{R}, \mathbf{C}, \mathbf{H}, \mathbf{O}$ in the construction and classification of, respectively, $N=1,2,4,8$ global supersymmetric quantum mechanical and classical dynamical systems. In particular an $N=8$ Malcev superaffine algebra is introduced and its relation to the non-associative $N=8 \mathrm{SCA}$ is discussed. A list of present and possible future applications is given.

\section{INTRODUCTION}

The investigation of extended supersymmetries in quantum mechanical systems is an essential tool for any realistic application of supersymmetry. Many examples can be given. Here I limit myself just to mention that any supersymmetric field theory in the ordinary Minkowski space, when dimensionally reduced to a one-temporal dimension [1], acquires 4 times the number of the original supersymmetries. For instance $N=$ 1,2, 4 super-YangMills theories are reduced to $N=4,8,16$ super quantum mechanical systems respectively.

Due to the lack of an explicit superfield formalism for large $N(N>4)$, such systems have received little attention in the literature, apart some specific cases, see e.g. [2] and [3].

On the other hand, since at least the work of Kugo and Townsend [4], it is known that the division algebras are related with the $N=1,2,4,8$ extended supersymmetries. However, still nowadays, the connection between division algebras and extended supersymmetries has not been completely worked out. In several cases it has been made explicit; in many other cases however it is only implicit or just suggested. This is especially true for the division algebra of octonions (or octaves) since, being non-associative, is technically more difficult to handle.

The aim of this paper is to partly fill this gap, presenting some new constructions which illustrate and make explicit such a connection in a few new cases. The results here presented are extracted from three recent articles [5], [6] and [7]. Their content being more general, only the part concerning the role of division algebras is here discussed. Proofs and details can be found there.

More specifically, the points here discussed are the relation between octonions and a given real Clifford algebra associated to the representation of the $N=8$ supersymmetric quantum mechanics algebra, as well as the explicit construction of the global $N=8$ supersymmetry in terms of charges derived from a super-current algebra which turns out to be a Malcev algebra (i.e. a special type of non-jacobian algebra). An algebraic interpretation (via Sugawara costruction) for the so-called non-associative $N=8$ superconformal algebra introduced in [8] is obtained as an extra bonus.

The above construction finds an immediate application in the extended supersymmetrization of integrable equations in $1+1$ dimensions (such as $\mathrm{KdV}$ ) [7]. A list of possible other applications is given in the Conclusions.

\section{DIVISION ALGEBRAS AND EX- TENDED SUPERSYMMETRIES}

Let me recall some basic properties of the division algebras $\mathbf{R}, \mathbf{C}, \mathbf{H}, \mathbf{O}$ and of their relation with extended supersymmetries.

Division algebras over the real field $\mathbf{R}$ can be obtained by iterating the "doubling" procedure (see 91) which allows to construct complex numbers out of real numbers. 
After each doubling some properties are lost. Quaternions are non-commutative, while octonions are non-associative. They satisfy however the weaker condition of alternativity (i.e. the associator $[a, b, c] \equiv(a b) c-a(b c)$ vanishes for $a=b)$.

By virtue of the generalized Frobenius theorem, $\mathbf{R}, \mathbf{C}, \mathbf{H}, \mathbf{O}$ are the only finite, alternative algebras over the real field up to isomorphism. In this respect they are a very fundamental and peculiar mathematical structure.

They can be used to construct an explicit realization of the one-dimensional supersymmetry algebra

$\left\{Q_{i}, Q_{j}\right\}=\delta_{i j} H$,

where the total number of supersymmetry charges $Q_{i}$ is $1,2,4$ or 8 respectively.

The relation can be made precise as follows. Let $\tau_{a}$ denote the imaginary octonions (for $a=$ $1,2, \ldots, 7$, while imaginary quaternions are restricted to the values $a=1,2,3$, and the imaginary unit is assumed to be given for $a=1$ ).

Explicitly, their multiplication rule can be presented through

$\tau_{a} \cdot \tau_{b}=-\delta_{a b} \mathbf{1}+C_{a b c} \tau_{c}$,

where the structure constants $C_{a b c}$ can be assumed to be totally antisymmetric and nonvanishing only for the special combinations

$a b c \equiv 123,147,165,246,257,354,367$

$\left(C_{a b c}\right.$ is normalized to 1 for even permutations of the above orderings).

If we introduce an $N=1$ one-dimensional superspace with bosonic coordinate $x$ and Grassmann variable $\theta$, we can easily construct an $(N=$ $2,4,8$ ) extended version of the supersymmetry algebra in terms of the generators

$Q_{0} \equiv Q=\frac{\partial}{\partial \theta}+\theta \frac{\partial}{\partial x}$,

while

$Q_{a} \equiv \tau_{a} D$,

with

$D=\frac{\partial}{\partial \theta}-\theta \frac{\partial}{\partial x}$, (here $i \equiv 0, a$ ). The introduction of imaginary octonions has been used to "correct" the sign appearing in the anticommutation of the fermionic derivative.

\section{3. $N=8$ REPRESENTATION MULTI- PLETS AND OCTONIONS}

Being non-associative, octonions cannot of course be represented through matrices with the standard product. On the other hand it is wellknown that octonions and pseudo-octonions are related to certain real representations of the Clifford algebras $C(p, q)$ (i.e. for a $(p, q)$ signature), such as $C(0,7), C(0,8)$ and $C(8,0)$. An explicit construction is given in 10 .

The 16-dimensional matrix representations $C(8,0)$ and $C(0,8)$ are of particular interest in the light of the results of [5]. In that paper some properties of the finite irreducible multiplets of representation of the one-dimensional $\mathrm{N}$-extended supersymmetry have been proven. The results can be summarized as follows. At first it is shown that any such multiplet contains an equal number (say $d$ ) of bosons and fermions distributed in different spin states. Any given multiplet under consideration can be unambiguously "shortened" to a standard (fundamental) multiplet such that all $d$ bosons and all $d$ fermions are grouped in just two spin states (one for the bosons, the other one for the fermions). Since all irreducible representations are specified by the given irreducible fundamental multiplet, which can be regarded as both the generator and the representative of its class, it is of tantamount importance to classify all classes of irreducible fundamental multiplets. The answer can be given rather easily. It turns out that they are in one-to-one correspondence with the real representations of Clifford algebras which are of Weyl form, i.e. such that all matrices $\Gamma_{i}$ can be put in a block antidiagonal form, $\Gamma_{i}=\left(\begin{array}{cc}0 & \sigma_{i} \\ \tilde{\sigma}_{i} & 0\end{array}\right)$, for $i=1, \ldots, N$. The correspondence is such that the space-dimensionality coincides with $N$, the number of extended supersymmetries, while the dimensionality of the $\Gamma_{i}$ matrices is $2 d$, the total number of bosons and fermions. 
The reality condition imposed on the $\Gamma_{i}$ is in consequence of the reality property of the bosonic and fermionic fields, while the Weyl property can be understood by loosely stating that the Clifford's $\Gamma_{i}$ matrices are now promoted to be supermatrices which act on and exchange the fermionic and the bosonic subspaces.

The classification of all finite real representations of Clifford algebras, satisfying the further Weyl property, has been given in the literature in several places (see [5] for references).

While the construction in [5] has been performed independently of and without any mention to division algebras, just for the special case of $C(0,8)$ and $C(8,0)$, something more can be said. They are both Weyl-type representations which are associated with the 16-dimensional (8 bosons and 8 fermions) irreducible fundamental multiplet of the $N=8$ supersymmetry. It is therefore possible to combine the results of [5] with the relation, presented in [10], on the connections with such real Clifford representations and the octonions. The final result consists in the established connection between a matrix representation of the global $N=8$ supersymmetry, and the $N=8$ octonionic construction which has been discussed in the previous section.

\section{SUPERAFFINE ALGEBRAS OVER DIVISION ALGEBRAS}

In the previous section we have seen that there are two related ways of implementing an $N=8$ global supersymmetry. In terms of a matrix representation or by making use of the octonions. In this section I discuss under which conditions the octonionic-realized $N=8$ global supersymmetry can be recovered from charges of an associated supercurrent algebra. More generally, I discuss how it is possible to "superaffinize" a division algebra, following [6] .

There exists a well-defined procedure to (super)affinize any given simple Lie or super-Lie algebra $\mathcal{G}$. The details are given e.g. in [1], where some physical applications are also discussed. No matter which is the starting (super)Lie algebra $\mathcal{G}$, the associated superaffine algebra is an ordinary superLie algebra which in particular satisfies the superJacobi identity. A drastically new feature arises when the starting algebra is the division algebra of octonions. Due to the alternative property, the commutator algebra of octonions

$\left[\tau_{a}, \tau_{b}\right]=f_{a b c} \tau_{c}$

(with $f_{a b c}=2 C_{a b c}$, the structure constants introduced in section 2) no longer satisfies the Jacobi identity, but the weaker condition

$J(x, y,[x, z])=[J(x, y, z), x]$,

which, together with $[x, x]=0$, gives it the structure of a Malcev algebra (the property (8) holds for any three given elements $x, y, z$, where $J(x, y, z)$ is the jacobian).

Let us consider the algebra of commutators satisfied by the seven imaginary octonions plus the identity 1 which, for our scopes, can also be expressed as $\tau_{0}$. It admits a quaternionic subalgebra which coincides with the $s l(2) \oplus u(1)$ Lie algebra. The superaffinization requires at first the construction of a superloop algebra. This implies to associate to any bosonic generator $\tau_{i}$ a corresponding fermionic $N=1$ superfield $\Psi_{i}(X)=$ $\psi_{i}(x)+\theta j_{i}(x)$. Let us denote with $f_{i j k}$ the original structure constants $f_{i j k}$, the superloop algebra can be introduced through

$\left\{\Psi_{i}\left(X_{1}\right), \Psi_{j}\left(X_{2}\right)\right\}=f_{i j k} \Psi_{k}\left(X_{2}\right) \delta\left(X_{1}, X_{2}\right)$,

(where $\delta\left(X_{1}, X_{2}\right) \equiv \delta\left(x_{1}-x_{2}\right)\left(\theta_{1}-\theta_{2}\right)$ is the supersymmetric delta-function). Next, in order to affinize the superloop algebra, a central extension $k$ must be introduced in a manifestly supersymmetric form. In the case of a Lie algebra (such as the $\operatorname{sl}(2) \oplus u(1)$ subalgebra) a central extension appearing in the r.h.s. of (9) takes the form

$k \cdot \operatorname{tr}\left(\tau_{i} \tau_{j}\right) D_{X_{2}} \delta\left(X_{1}, X_{2}\right)$,

where $D$ is the $N=1$ fermionic derivative (6), while the trace is computed on some matrix representation for $\tau_{i}, \tau_{j}$. In the case of octonions, due to the obvious absence of matrix representations, the above formula is meaningless and must be replaced by some generalization. It turns out that the correct generalization is given by

$k \cdot \Pi\left(\tau_{i} \cdot \tau_{j}\right) D_{X_{2}} \delta\left(X_{1}, X_{2}\right)$, 
where $\Pi\left(\tau_{i} \cdot \tau_{j}\right)$ corresponds to the projection over the identity 1 . On the quaternionic subalgebra it reproduces the correct formula for the central extension, as it should be.

The affinization of (the commutator of) the division algebra of the octonions has been considered in a series of papers by Osipov, two of them [12] devoted to the bosonic affine case and its related Sugawara construction, while the third one [13] to construct the superaffine algebra. While in the bosonic subalgebra case (setting all fermionic fields equal to zero) our results coincide with the Osipov's results, the central extension presented in 13. is not manifestly supersymmetric, as an easy inspection shows. The central extension (11) given here, on the contrary, is manifestly supersymmetric. We therefore regard the (9) loop algebra, centrally extended through (11) as the correct superaffinization of the commutator algebra of the octonions. A first property can be immediately stated, namely that the superaffine octonionic algebra is a superMalcev algebra, satisfying a graded version of the Malcev property, which is expressed in terms of the superjacobian.

Despite the fact that the superaffine algebra here introduced is expressed in terms of manifestly $N=1$ superfields only, its association with a division algebra suggests that it possesses extra supersymmetries. This is indeed the case. It is in fact a global $N=8$ supersymmetric algebra. The sketch of the proof, following [6], will be given in the next section, where the supersymmetric Sugawara construction will be discussed. Let us conclude this section by mentioning that the restriction to the quaternionic case, once expressed in component fields, corresponds to the $N=4$ superaffine algebra introduced in [14], in terms of a manifest $N=2$ superfields formalism. The division algebra properties were assumed, but no explicit use of them was made in that construction.

\section{THE SUGAWARA CONSTRUCTION FOR THE NON-ASSOCIATIVE $N=8$ SCA}

The superaffine algebra introduced in the previous section, which can be denoted as the $\hat{\mathbf{O}}$ - superalgebra, is $N=8$, as already recalled. An explicit way to prove it consists in constructing a Sugawara realization which produces an $N=8$ extension of the Virasoro algebra. This has been done in [6] by using an improved version (to deal with octonions) of the Thieleman's Mathematica package for OPE's computations. The $N=8$ extension of Virasoro coincides with the $N=8$ (so-called non-associative) Superconformal algebra introduced in [8], which is some sort of minimal $N=8$ extension of Virasoro, being generated, besides the Virasoro field $T$, by 8 spin- $\frac{3}{2}$ fermionic fields $Q_{i}$ and 7 spin- 1 bosonic currents $J_{a}$. It is constructed with the structure constants of the octonions and for that reason the Jacobi identities are not satisfied.

The Sugawara construction requires the notion of the enveloping algebra. It is worth stressing the fact that in the case of the superMalcev $\mathbf{O}$ algebra the bosonic and fermionic component fields are assumed to be real-valued (and not octonionicvalued). The $\hat{\mathbf{O}}$ algebra must therefore be regarded as an abstract non-Lie algebra of PoissonMalcev brackets. When considering the enveloping algebra the Poisson-Malcev brackets are assumed to satisfy the graded version of the Leibniz rule. One important remark is the following, the Malcev property is not closed under composition law, i.e., even if the triples $x_{1}, y, z$ and $x_{2}, y, z$ both satisfy (8), it is not guaranteed that the triple $x=x_{1} x_{2}, y, z$ is Malcev. Indeed, the $N=8$ non-associative SCA is not super-Malcev since, e.g., the (8) property is violated for $J_{1}, J_{2}, Q_{4}$, while its bosonic subalgebra is of Malcev type.

For what concerns the Sugawara construction of $\hat{\mathbf{O}}$, two distinct limiting subcases have to be distinguished. The purely bosonic limit, obtained by disregarding all fermionic fields, which reproduces the results obtained in [12], and the quaternionic case, obtained by disregarding the fields associated to the values $i=4,5,6,7$. It corresponds to the Sugawara construction of [14], which reproduces the minimal $N=4$ Superconformal algebra. Such a Sugawara realizes an interpolation between the super-NLS and the super-mKdV hierarchies.

Since the details of the construction and the full 
list of formulas can be found in [6], here I limit myself to stress the salient points. Unlike the bosonic and the $N=4$ subcases, the construction of the $N=8$ Sugawara requires a classical renormalization procedure and is recovered only in the limit $k \rightarrow \infty$, where $k$ is the central charge (of the superaffine algebra). The reason is due to the fact that the algebra of the Sugawara-constructed fields does not close automatically on the nonassociative $N=8 \mathrm{SCA}$, since extra-contributions, proportional to the fermionic fields $\psi_{i}(x)$, for $i=4,5,6,7$, appear. These extra-contributions however disappear in the limit $k \rightarrow \infty$. A simultaneous rescaling (proportional to $k$ ) of the Poisson-Malcev brackets normalization is needed in order to produce a finite value of the central extension $c$ of the $N=8 \mathrm{SCA}$. Due to the presence of this "classical renormalization", no relation is found between the affine central charge $k$ and the conformal central charge $c$. The latter, with a finite rescaling of the fields and of the brackets, can be normalized at will. Taking the $k \rightarrow \infty$ limit is safe since the terms which disappear in the r.h.s. are always of higher order in $\epsilon=\frac{1}{k}$ w.r.t. the surviving terms which close the $N=8$ SCA.

To illustrate the above points I present here the form of the Sugawara just for the Virasoro field $T(x)$ and the spin- $\frac{3}{2}$ field $Q(x)$ associated to the identity $\mathbf{1}$. We have (the convention over repeated indices is understood)

$$
\begin{aligned}
T= & \frac{1}{k^{2}}\left(j_{i} j_{i}+\psi_{i}{ }^{\prime} \psi_{i}\right)+\frac{1}{k} j_{0}{ }^{\prime}- \\
& \frac{2}{3 k^{3}} C_{a b c} \psi_{a} \psi_{b} j_{c}-\frac{2}{k^{4}} C_{a b c d} \psi_{a} \psi_{b} \psi_{c} \psi_{d}, \\
Q= & \frac{1}{k^{2}} \psi_{i} j_{i}+\frac{1}{k} \psi_{0}{ }^{\prime}-\frac{2}{3 k^{3}} C_{a b c} \psi_{a} \psi_{b} \psi_{c},
\end{aligned}
$$

where the dual tensor (in the seven-dimensional imaginary octonionic space) $C_{a b c d}=\epsilon_{a b c d e f g} C_{e f g}$ is totally antisymmetric.

While the Virasoro algebra for $T(x)$ is satisfied exactly, the spin- $\frac{3}{2}$ primary condition for the $Q_{0}(x) \equiv Q(x)$ field is guaranteed only in the $k \rightarrow \infty$ limit, indeed

$$
\begin{aligned}
\{T(x), T(y)\}_{R} & =-\frac{1}{2} \delta^{\prime \prime \prime}+2 T(y) \delta^{\prime}+T(y)^{\prime} \delta, \\
\{T x), Q(y)\}_{R} & =\frac{3}{2} Q(y) \delta^{\prime}+Q(y)^{\prime} \delta+X_{1},
\end{aligned}
$$

$\{Q(x), Q(y)\}_{R}=-\frac{1}{6} \delta^{\prime \prime \prime}+\frac{1}{2} T(y) \delta+X_{2}$,

(in the above formulas $\delta \equiv \delta(x-y)$ and the derivative is taken in $y$ ). The extra-terms $X_{1}, X_{2}$ are given by

$$
\begin{aligned}
& X_{1}=-\frac{3}{2 k^{4}} C_{a b c d} \psi_{a} \psi_{b} \psi_{c} j_{d} \delta, \\
& X_{2}=-\frac{5}{2 k^{4}} C_{a b c d} \psi_{a} \psi_{b} \psi_{c} \psi_{d} \delta .
\end{aligned}
$$

Notice that the extra-terms in the r.h.s. are not present when the Sugawara is restricted to the superaffinization of the quaternionic subalgebra or in the purely bosonic case.

Relations like those here shown hold for the whole set of fields which satisfy the $N=8$ nonassociative superconformal algebra.

By taking the Poisson brackets between the supersymmetric charges $Q_{i}=\int d x Q_{i}(x)$ and the original affine fields in $\hat{\mathbf{O}}$, we can recover in the $k \rightarrow \infty$ case the $N=8$ "octonionic" transformations (4) and (5), assumed to act on the octonionic-valued superfield $\Psi_{i}(X) \tau_{i}$. We have, e.g.,

$$
\begin{aligned}
\delta_{Q_{1}} j_{1} & =\frac{1}{2} \psi_{0}{ }^{\prime}+\frac{2}{k^{2}}\left(C_{a b c} \psi_{a} \psi_{b} \psi_{c}\right), \\
\delta_{Q_{1}} \psi_{1} & =-\frac{1}{2} j_{0} \\
\delta_{Q_{1}} j_{2} & =\frac{1}{2} \psi_{3}{ }^{\prime}+O\left(\frac{1}{k}\right), \\
\delta_{Q_{1}} \psi_{2} & =-\frac{1}{2} j_{3}+\frac{1}{k}\left(\psi_{1} \psi_{2}-\psi_{0} \psi_{3}\right) .
\end{aligned}
$$

(in the third equation the term $O\left(\frac{1}{k}\right)$ is rather complicated and is not necessary to report it here). Analogous results are found for the whole set of supersymmetric transformations $\delta_{Q_{i}}$ acting on the fields $\psi_{k}(x), j_{k}(x)$. Due to the "democratic" nature of the octonions (they are all on equal footing), the most general supersymmetry transformation can be directly read from (15).

The global $N=8$ octonionic transformation is interpreted, as promised, in terms of a supercurrent algebra. 


\section{AN APPLICATION: DIVISION AL- GEBRAS AND THE $N$-EXTENDED SUPERKdV}

From a practical point of view one of the most interesting features of the non-associative $N=8$ SCA is the presence of the central charge. The fact that this superalgebra does not satisfy the (super)Jacobi property allows to overcome the no-go theorem which states that central charges are not allowed for superconformal algebras with $N>4$. This is the case for standard superalgebras which satisfy the (super)Jacobi property. On the other hand the presence of a central charge is required if the algebra under consideration has to be interpreted as a Poisson-bracket algebra for a related KdV-type hamiltonian equation (the Virasoro central charge $\delta^{\prime \prime \prime}$ produces the $T^{\prime \prime \prime}$ term in the usual $\dot{T}=T^{\prime \prime \prime}+T T^{\prime} \mathrm{KdV}$ equation). It is therefore interesting to explore the possibility that the superaffine division algebra $\hat{\mathbf{O}}$ (and its subalgebras) could be related to supersymmetric extensions of $\mathrm{KdV}$, generating a hamiltonian dynamics. This possibility has been systematically investigated in [7], with a heavy use of computer algebraic manipulations. Leaving aside for the moment all questions related with the integrability of the extended system, in [7] the most general $N=4$ and $N=8$ hamiltonian supersymmetric extensions of $\mathrm{KdV}$, based on the $N=4$ minimal SCA and $N=8$ non-associative SCA respectively, have been constructed. The complete solution is the following. The most general $N=4$ supersymmetric hamiltonian for the $N=4 \mathrm{KdV}$ depends on 5 parameters (plus an overall normalization constant). However, if the hamiltonian is further assumed to be invariant under the involutions of the $N=4$ minimal SCA, three of the parameters have to be set equal to 0 . I recall here that the involutions of the $N=4$ minimal SCA are induced by the involutions of the quaternionic algebra. Three such involutions exist (any two of them can be assumed as generators), the $a$-th one (for $a=1,2,3$ ) is given by leaving $\tau_{a}$ (together with the identity) invariant and flipping the sign of the two remaining $\tau$ 's.

What is left is the most general hamiltonian, invariant under $N=4$ and the involutions of the algebra. It is given by

$$
\begin{aligned}
H_{2}= & T^{2}+Q_{i}^{\prime} Q_{i}-J_{a}^{\prime \prime} J_{a}+x_{a} T J_{a}{ }^{2}+ \\
& 2 x_{a} Q_{0} Q_{a} J_{a}-\epsilon_{a b c} x_{a} J_{a} Q_{b} Q_{c}+ \\
& 2 x_{1} J_{1} J_{2}^{\prime} J_{3}-2 x_{2} J_{1}^{\prime} J_{2} J_{3}
\end{aligned}
$$

(the convention over repeated indices is understood; here $i=0,1,2,3$ and $a=1,2,3)$.

The $N=4$ global supersymmetry requires the three parameters $x_{a}$ to satisfy the condition

$x_{1}+x_{2}+x_{3}=0$,

so that only two of them are independent. Since any two of them, at will, can be plotted in a real $x-y$ plane, it can be proven that the fundamental domain of the moduli space of inequivalent $N=4$ $\mathrm{KdV}$ equations can be chosen to be the region of the plane comprised between the real axis $y=0$ and the $y=x$ line (boundaries included). There are five other regions of the plane (all such regions are related by an $S_{3}$-group transformation) which could be equally well chosen as fundamental domain.

In the region of our choice the $y=x$ line corresponds to an extra global $U(1)$-invariance, while the origin, for $x_{1}=x_{2}=x_{3}=0$, is the most symmetric point (it corresponds to a global $S U(2)$ invariance).

The involutions associated to each given imaginary quaternion allows to consistently reduce the $N=4 \mathrm{KdV}$ equation to an $N=2 \mathrm{KdV}$, by setting simultaneously equal to 0 all the fields associated with the $\tau$ 's which flip the sign, e.g. the fields $J_{2}=J_{3}=Q_{2}=Q_{3}=0$ for the first involution (and similarly for the other couples of values 1,3 and 1,2). After such a reduction we recover the $N=2 \mathrm{KdV}$ equation depending on the free parameter $x_{1}$ (or, respectively, $x_{2}$ and $x_{3}$ ).

The integrability is known for $N=2 \mathrm{KdV}$ to be ensured for three specific values $a=-2,1,4$, discovered by Mathieu [15], of the free parameter $a$. We are therefore in the position to determine for which points of the fundamental domain the $N=4 \mathrm{KdV}$ is mapped, after any reduction, to one of the three Mathieu's integrable $N=2$ KdVs. It turns out that in the fundamental domain only two such points exist. Both of them lie on the $y=x$ line. One of them produces, after inequivalent $N=2$ reductions, the $a=-2$ and the 
$a=4 N=2 \mathrm{KdV}$ hierarchies. The second point, which produces the $a=1$ and the $a=-2 N=2$ $\mathrm{KdV}$ equations, however, does not admit at the next order an $N=4$ hamiltonian which is in involution w.r.t. $H_{2}$. In this respect the results in [7], which can be regarded as more general since are based on an exhaustive component-fields analysis, are in agreement with those of [16], based on an extended superfield formalism.

The next step considered in [7] was to perform a similar analysis for the $N=8$ case based on the $N=8$ non-associative SCA. At first the most general hamiltonian with the right dimension has been written down. Later, some constraints on it have been imposed. The first set of constraints requires the invariance under all the 7 involutions of the algebra. In the case of octonions the total number of involutions is 7 (with 3 generators) each one being associated to one of the seven combinations appearing in (3)). In the case, e.g., of 123 the corresponding $\tau_{a}$ 's are left invariant, while the remaining four $\tau$ 's, living in the complement, have the sign flipped.

The second set of constraints requires the invariance under the whole set of $N=8$ global supersymmetries. Under this condition there exists only one hamiltonian, up to the normalization factor, which is $N=8$ invariant. It does not contain any free parameter and is quadratic in the fields. It is explicitly given by

$H_{2}=T^{2}+Q_{i}^{\prime} Q_{i}-J_{a}^{\prime \prime} J_{a}$

(here $i=0,1, \ldots, 7$ and $a=1,2, \ldots, 7$ ).

The hamiltonian corresponds to the origin of coordinates (confront the previous case) which is also, just like the $N=4$ case, the point of maximal symmetry. This means that the hamiltonian is invariant under the whole set of seven global charges $\int d x J_{a}(x)$, obtained by integrating the currents $J_{a}$ 's.

Despite its apparent simplicity, it gives an $N=$ 8 extension of $\mathrm{KdV}$ which does not reduce (for any $N=2$ reduction) to the three Mathieu's values for integrability. Nevertheless the main result, highly non-trivial, consists in the proof of the existence of an $N=8$ extension of the $\mathrm{KdV}$ equation whose dynamics is hamiltonian (for a generalized non-Lie Poisson-brackets structure).
As a matter of fact, requiring the invariance under $N=5$ supersymmetries already uniquely specifies (18), since the remaining supersymmetries are generated by the previous ones. $N=4$ is the maximal supersymmetry which allows the presence of a global parameter. Two inequivalent ways of determining a free parameter-dependent $N=4$-invariant second hamiltonian $\mathrm{H}_{2}$, constructed with the $N=8 \mathrm{SCA}$ fields, are allowed. The first one requires $N=4$ invariance w.r.t. the $N=4$ global supercharges constructed from the $N=4$ minimal SCA subalgebra. The second one requires the invariance w.r.t. the remaining 4 supersymmetric generators. The explicit formulas for such $\mathrm{H}_{2}$ 's and the derived hamiltonian equations are presented in [7].

\section{CONCLUSIONS}

In this paper I have presented some new results concerning an explicit connection of extended supersymmetries and division algebras. Besides the relation between matrix and octonionic representations of the global $N=8$ supersymmetry, the superaffinization of a Malcev algebra based on octonions has been introduced, as well as its $N=8$ Sugawara construction which links it to the nonassociative $N=8 \mathrm{SCA}$ of reference [8].

As an application, the role of such algebras in furnishing a generalized Poisson bracket structure for the hamiltonian dynamics of supersymmetric extensions of the KdV equation has been examined. It has been proven, following [7], the existence of a unique $N=8 \mathrm{KdV}$ equation based on the $N=8$ non-associative SCA. Many more applications are likely to be studied with the tools here discussed. A list of possible topics can be furnished. In the light of the geometric approach, see [17], it is likely that the dynamics of a classical superstring moving in a flat target could be recovered from a supersymmetric version of the Liouville equation. If the target is 3 -dimensional, this is indeed the case. For 4 dimensional, 6 dimensional and 10-dimensional targets the corresponding super-Liouville theory is expected to take values in the complex, quaternionic and octonionic division algebras respectively. The algebraic structures here discussed seem the most 
natural framework to investigate such questions. On the other hand, either WZNW-type of models defined on the "almost group manifold" $S^{7}$ (the almost being referred to the fact that it can be recovered from imaginary octonions, together with their product which, however, is non-associative), or the dynamics of generalized tops moving on the $S^{7}$ sphere, are likely to be described by Malcev algebras. A converse approach, which retains Jacobi identities at the price of making fielddependent the algebraic structure constants (soft algebras) has been suggested in 18 .

Another natural setting concerns the application of such formalism to the twistors in investigating the quantization of the Green-Schwarz string [19].

\section{Acknowledgments}

I am grateful to the organizers of the Kharkov International Conference "Supersymmetry and Quantum Field Theory" for their kind invitation. It is a pleasure to thank my collaborators A. Pashnev from Dubna and H.L. Carrion and M. Rojas from CBPF.

\section{REFERENCES}

1. M. Claudson and M.B. Halpern, Nucl. Phys. B 250 (1985) 689.

2. R.A. Coles and G. Papadopoulos, Class. Quant. Grav. 7 (1990) 427.

3. S.J. Gates, Jr and L. Rana, Phys. Lett. B 352 (1995); ibid. B 369 (1996) 262.

4. T. Kugo and P. Townsend, Nucl. Phys. B 221 (1983) 357.

5. A. Pashnev and F. Toppan, "On the Classification of $N$-extended Supersymmetric Quantum Mechanical Systems", preprint CBPFNF-029/00, JINR E2-2000-193, in hepth/0010135.

6. H.L. Carrion, M. Rojas and F. Toppan, "An $N=8$ Superaffine Malcev Algebra and Its $N=8$ Sugawara", preprint CBPF-NF011/01.

7. H.L. Carrion, M. Rojas and F. Toppan, "Division Algebras and the $N=1,2,4,8 \mathrm{Su}-$ persymmetric Extensions of KdV", preprint CBPF-NF-012/01.

8. F. Englert, A. Sevrin, W. Troost, A. van
Proeyen and P. Spindel, Jou. Math. Phys. 29 (1988) 281.

9. M. Postnikov, "Lie Groups and Lie Algebras", Moscow, Nauka (Eng. version) (1982).

10. S. Okubo, Jou. Math. Phys. 32 (1991) 1657; ibid. 1669 .

11. F. Toppan, in Supersymmetry and Integrable Models, Lect. Notes in Phys. 502, SpringerVerlag, Berlin (1998) 283.

12. E.P. Osipov, Phys. Lett. B 214 (1988) 371; Lett. Math. Phys. 18 (1989) 35.

13. E.P. Osipov, Phys. Lett. B 274 (1992) 341.

14. E. Ivanov, S. Krivonos and F. Toppan, Phys. Lett. B 405 (1997) 85.

15. P. Mathieu, Phys. Lett. B 203 (1988) 287.

16. F. Delduc and E. Ivanov, Phys. Lett. B 309 (1993) 312.

17. I. Bandos, D. Sorokin and D. Volkov, Phys. Lett. B 372 (1996) 77.

18. M. Cederwall and C. Preitschopf, Comm. Math. Phys. 167 (1995) 373.

19. N. Berkovits, Nucl. Phys. B 358 (1991) 169. 